\title{
Vibration Characteristic of a Transition Constrained Damping Beam Based on the Shear Dissipating Energy Assumption
}

\author{
Bijuan Yan, Huijun Liang and Minjie Jin \\ School of Mechanical Engineering, Taiyuan University of Science and Technology, Taiyuan 030024, China.
}

\begin{abstract}
(Received 19 July 2018; accepted 22 November 2018)
Adding a "transition layer" could further improve the dissipation ability of the original constrained damping structure to the external vibration. At the same time, the addition of the transition layer brings many difficulties in establishing the model and acquiring the relevant characteristics of the structure. Based on the shear dissipating energy assumption and the Hamilton principle, the finite element model of the transition constrained damping beam is established. On the basis of this reasonable assumption, the whole derivation process is simplified and easy to read by regularizing the element stiffness and mass matrix, and the expression of loss factor and natural frequency of damping beam is obtained. In order to verify the correctness of the model, the computed results are compared with the analytical solution, and both are found to be in good agreement. Taking the cantilever damping beam as an example, the influence of the material choice of the transition layer and the structural parameters on the natural frequency and the loss factor of the structure are discussed. The results of this paper would lay a good foundation for further optimization and practical engineering application.
\end{abstract}

\section{INTRODUCTION}

The traditional three-layer constrained damping beam mainly depends on the longitudinal shear and transverse tensile deformation of viscoelastic damping material to dissipate the external vibration energy. ${ }^{1}$ However, its damping effect in practical engineering applications is often limited to a certain extent. To solve this problem, researchers from all over the world have presented some methods from different aspects. For example, Lepoittevin et al. designed the outermost constrained layer with some segments, and the segment numbers and distribution were analysed. ${ }^{2}$ Kumar et al. used the piezoelectric material to replace the traditional metal materials of constrained layer, so the active control was applied into the sandwich structure plate to improve of the energy dissipation effect of the whole structure. ${ }^{3}$ In addition, a transition constrained damping structure was presented, in which the "transition layer" was added between the base and viscoelastic layers, the related results showed that the longitudinal shear deformation of the viscoelastic layer could be further increased by adding the transition layer, and thus the desired damping characteristics was achieved. ${ }^{4}$ Figure 1 shows the structure of transition constrained damping beam (TCDB).

At present, there are two hypotheses about the energy dissipation of damping structure: one is shear deformation and the other is tensile deformation. However, which occupies dominant position is still controversial. According to the related literature, most of the studies employed the shear deformation hypothesis. For shear deformation, the analytical method and finite element method are usually used as the main analysis means. ${ }^{5}$ For example, Kerwin established the theoretical model of a three-layer beam based on the complex modulus method for the first time; Ditaranto considered the shear effect of a viscoelastic layer and established the sixth- order differential equation of a passive constrained damping beam (PCLD) ${ }^{6,7}$ Thereafter, Rao analysed the natural frequency and the loss factor of the PCLD beam under different boundary conditions. ${ }^{8}$ The semi-analytical solution of a threelayer damping structure was obtained by Tang et al., using the strip transfer function method. ${ }^{9}$ Although the exact solution of a damping structure can be obtained theoretically, the general solution of high-order differential equation is rather difficult, and its solution depends on simple structure and special boundary conditions, which leads to some limitations in engineering application. ${ }^{10}$ To overcome these shortcomings, the finite element method is used to analyse the related characteristics of damping structures. For example, a finite element model of three-layer sandwich structure was established for the dynamic analysis by Johnson et al.; Sainsbury et al. constructed a PCLD beam finite element model through the Galerkin orthogonal function to reduce the amount of calculation and improve the calculation efficiency. ${ }^{11,12}$ In addition, Deng et al. made the dynamic analysis of PCLD plate by using the GHM model. ${ }^{13}$ However, it is easy to find that the above-mentioned studies mainly focus on the three-layer constrained damping structure. For the transition constrained damping structure, Zhang et al. used the complex stiffness method to obtain the structure loss factor and discussed the influence of transition layer laying position. ${ }^{14}$ However, the solution of this method must be repeated many times, so it will increase the computational complexity of the whole calculation process. At the same time, this method can only get the loss factor of the structure, but the natural frequency of the structure cannot be obtained. In addition, that research gave the dynamic response of TCDB, but the results were effective only when the boundary conditions of TCDB was that the one end of the base layer was fixed, and the remaining layers remained free. ${ }^{4}$ And the research only 


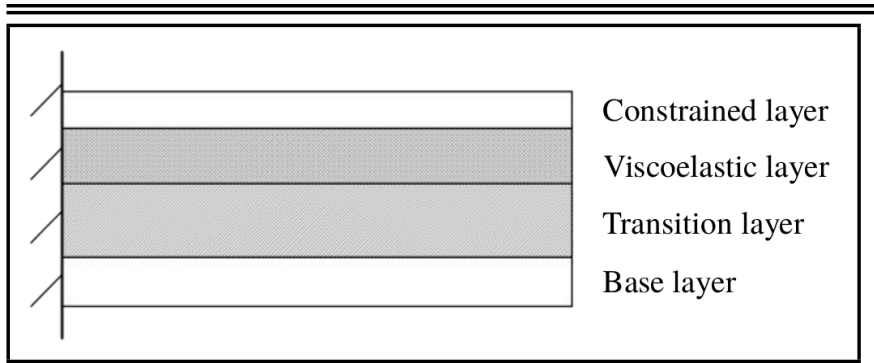

Figure 1. Schematic drawing of TCDB.

discussed the influence of transition layer parameters on the behaviour of the whole structure, but the rest parameters of the other layers were not discussed. ${ }^{4}$

In order to compensate the lack of the present research about the transition constrained damping beam, this current paper established the finite element model of TCDB. During the derivation process, the viscoelastic layer and transition layer are assumed to only produce the longitudinal shear deformation and the rotary inertia of each layer are ignored. However, due to the adding of the transition layer, it will bring about a lot of inconvenience of the whole formula derivation of the model, so this paper took the method of making the element stiffness and mass matrix become more regular in order that the whole expressions got more concise and clear. At the same time, the correctness of the model was verified, the shear modulus and material loss factor of transition layer were discussed, the influences of the thickness of each layer on the natural frequency and the loss factor of TCDB were also discussed. The results in this paper have a certain guiding significance to the application of the damping structure in actual engineering.

\section{FINITE ELEMENT MODEL OF TCDB}

\subsection{Model Assumptions}

In order to facilitate the construction of TCDB finite element model, this paper made the following assumptions: (1) the compression deformation along with the thickness of TCDB was neglected, that is to say, each layer had the same deflection; (2) the viscoelastic layer and transition layer only produced longitudinal shear deformation; (3) the effect of the moment of inertia of each layer was ignored; (4) each layer was pasted firmly, no relative sliding produces and the displacement between all layers was continuous; (5) the deformation was limited to the scope of linear viscoelasticity.

\subsection{Kinematics Relations}

The geometry and deformation relationship of TCDB is shown in Fig. 2. Here, the dotted line represents the neutral surface of each layer: $\partial w / \partial x$ was the rotation angle of the transition constrained damping beam, $\psi$ denoted the shear angle of the transition layer, $\gamma$ was the shear angle of the viscoelastic layer. The set $u_{i}(i=b, s, v, c)$ denoted the displacements of the base layer, transition layer, viscoelastic layer, and constrained layer in the $x$-direction. Respectively, $h_{i}(i=b, s, v, c)$ was the thicknesses of each layer.

According to Fig. 2 and the classic Euler Bernoulli beam theory, ${ }^{15}$ the displacement in the $x$-direction for the transition

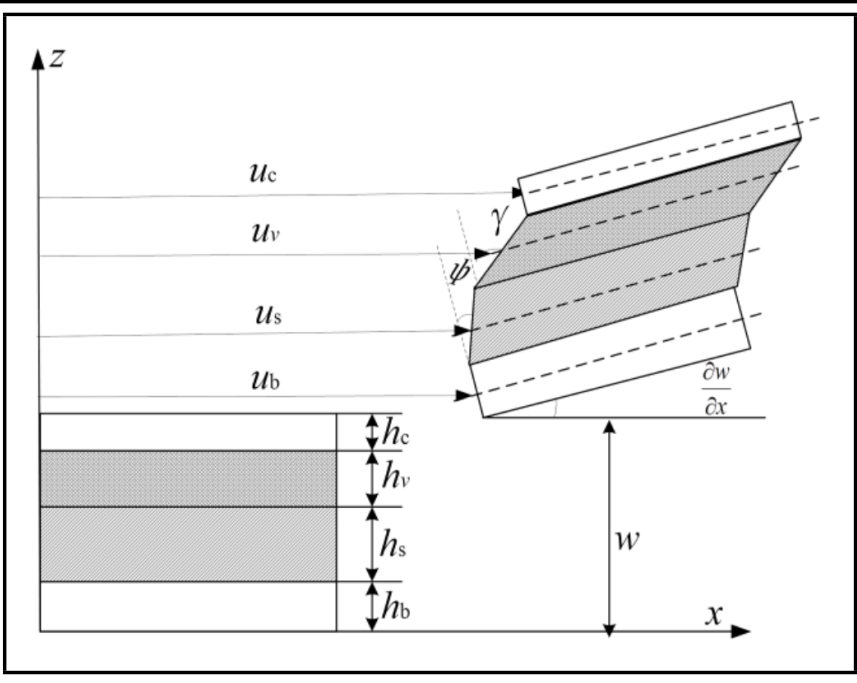

Figure 2. Geometric and deformation of TCDB.

layer and viscoelastic layer were given as follows:

$$
\left\{\begin{array}{l}
u_{s}=u_{b}-\left(\frac{h_{b}+h_{s}}{2}\right) \frac{\partial w}{\partial x} \\
u_{v}=u_{c}+\left(\frac{h_{v}+h_{c}}{2}\right) \frac{\partial w}{\partial x}
\end{array} .\right.
$$

Based on Hooke's law and the axial force balance condition of TCDB,${ }^{16,17}$ the shear angles of the transition layer and viscoelastic layer were expressed as:

$$
\left\{\begin{array}{r}
\gamma=\left[\frac{\left(u_{c}-u_{b}\right)}{h_{s}}+\left(1+\frac{h_{c}+2 h_{v}+2 y_{b}}{2 h_{s}}\right) \frac{\partial w}{\partial x}\right] \\
\cdot\left(\frac{1}{h_{v} / h_{s}+G_{v} / G_{s}}\right) \\
\psi=\left[\frac{\left(u_{c}-u_{b}\right)}{h_{s}}+\left(1+\frac{h_{c}+2 h_{v}+2 y_{b}}{2 h_{s}}\right) \frac{\partial w}{\partial x}\right] \\
\cdot\left(\frac{G_{v} / G_{s}}{h_{v} / h_{s}+G_{v} / G_{s}}\right)
\end{array}\right.
$$

where $y_{b}$ represented the distance from the upper surface of the constrained layer to the neutral surface of the TCDB. In general, it could be replaced by $h_{b} / 2$.

\subsection{Shape Function}

For the transition constrained damping beam, the beam element with a one-dimensional two node $(i, j)$ was used. The beam element length was denoted with $l$, each node had four degrees of freedom $\left(w, \theta, u_{c}, u_{b}\right)$, respectively. Among the four degrees of freedom, $w$ was the longitudinal displacement of TCDB (i. e. deflection), $\theta$ was the rotation angle of cross section, $u_{c}$ was the axial displacement of the constrained layer, and $u_{b}$ was the displacement of the base layer. The node displacement vector for beam element was as follows: ${ }^{10}$

$$
\left\{\Delta^{e}\right\}=\left\{\begin{array}{llllllll}
w_{i} & \theta_{i} & u_{c i} & u_{b i} & w_{j} & \theta_{j} & u_{c j} & u_{b j}
\end{array}\right\}^{T} .
$$

The deflection $w$ is denoted with the Hermite shape function, and the axial displacements of $u_{c}, u_{b}$ were given by the Lagrange shape function. Therefore, the shape function was as follows:

$$
\left\{\begin{array}{c}
w=a_{0}+a_{1} \xi+a_{2} \xi^{2}+a_{3} \xi^{3} \\
u_{b}=b_{0}+b_{1} \xi \\
u_{c}=c_{0}+c_{1} \xi
\end{array} ;\right.
$$


where $\xi=x / l$, and the displacement of each element in the beam element could be defined as:

$$
\{\Delta\}=\left[\begin{array}{llll}
w & \theta & u_{c} & u_{b}
\end{array}\right]^{T}=N\left\{\Delta^{e}\right\}
$$

where $N=\left[\begin{array}{llll}N_{w}^{T} & N_{\theta}^{T} & N_{u_{c}}^{T} & N_{u_{b}}^{T}\end{array}\right]^{T}, N$ was a $4 \times 8$ matrix and each component was defined as in $\mathrm{Eq}(6)$.

Therefore, the 4 degrees of freedom of the beam element were written in a shape function as follows:

$$
\begin{aligned}
w & =\left[N_{w}\right]\left\{\Delta^{e}\right\}, & \theta & =\left[N_{\theta}\right]\left\{\Delta^{e}\right\}, \\
u_{c} & =\left[N_{u_{c}}\right]\left\{\Delta^{e}\right\}, & u_{b} & =\left[N_{u_{b}}\right]\left\{\Delta^{e}\right\} .
\end{aligned}
$$

At the same time, the $x$-direction displacement and shear angle of the transition layer and viscoelastic layer could be expressed with the shape function as follows:

$$
\begin{aligned}
u_{s} & =\left[N_{s}\right]\left\{\Delta^{e}\right\}, & u_{v} & =\left[N_{v}\right]\left\{\Delta^{e}\right\}, \\
\psi & =\left[N_{\psi}\right]\left\{\Delta^{e}\right\}, & \gamma & =\left[N_{\gamma}\right]\left\{\Delta^{e}\right\} .
\end{aligned}
$$

In the equation (8), $N_{s}, N_{v}, N \psi, N \gamma$ were defined as follows:

$$
\begin{aligned}
N_{s} & =\left[N_{u_{b}}\right]-\left(\frac{h_{b}+h_{s}}{2}\right)\left[N_{\theta}\right] ; \\
N_{v} & =\left[N_{u_{c}}\right]+\left(\frac{h_{c}+h_{v}}{2}\right)\left[N_{\theta}\right] ; \\
N_{\psi} & =\left[\frac{\left(\left[N_{c}\right]-\left[N_{b}\right]\right)}{h_{s}}+\left(1+\frac{h_{c}+2 h_{v}+2 y_{b}}{2 h_{s}}\right)\left[N_{\theta}\right]\right] \\
& \cdot\left(\frac{G_{v} / G_{s}}{h_{v} / h_{s}+G_{v} / G_{s}}\right) ; \\
N_{\gamma} & =\left[\frac{\left(\left[N_{c}\right]-\left[N_{b}\right]\right)}{h_{s}}+\left(1+\frac{h_{c}+2 h_{v}+2 y_{b}}{2 h_{s}}\right)\left[N_{\theta}\right]\right] \\
& \cdot\left(\frac{1}{h_{v} / h_{s}+G_{v} / G_{s}}\right) .
\end{aligned}
$$

\subsection{Beam Element Stiffness Matrix}

The stiffness matrix of the beam element could be obtained by the energy method. The total potential energy $U$ of the beam element was equal to the sum of the bending potential $U_{b c}, U_{b b}$, tensile potential $U_{t c}, U_{t b}$ of the constraining layer and the base layer, and the shear potential $U_{s v}, U_{s s}$ of the viscoelastic layer and the transition layer. That is, the total potential energy $U$ of the beam element could be expressed as: ${ }^{10}$

$$
U=\left[U_{b c}\right]+\left[U_{t c}\right]+\left[U_{b b}\right]+\left[U_{t b}\right]+\left[U_{s v}\right]+\left[U_{s s}\right]
$$

where:

$$
\begin{aligned}
U_{b i} & =\frac{1}{2} E_{i} I_{i} \int_{0}^{l}\left(\frac{\partial^{2} w}{\partial x^{2}}\right)^{2} d x=\frac{1}{2}\left\{\Delta^{e}\right\}^{T}\left[K_{b i}\right]\left\{\Delta^{e}\right\} \\
U_{t i} & =\frac{1}{2} E_{i} A_{i} \int_{0}^{l}\left(\frac{\partial u_{i}}{\partial x}\right)^{2} d x=\frac{1}{2}\left\{\Delta^{e}\right\}^{T}\left[K_{t i}\right]\left\{\Delta^{e}\right\} \\
U_{s s} & =\frac{1}{2} G_{s} A_{s} \int_{0}^{l} \psi^{2} d x=\frac{1}{2}\left\{\Delta^{e}\right\}^{T}\left[K_{s}\right]\left\{\Delta^{e}\right\} \\
U_{s v} & =\frac{1}{2} G_{v} A_{v} \int_{0}^{l} \gamma^{2} d x=\frac{1}{2}\left\{\Delta^{e}\right\}^{T}\left[K_{v}\right]\left\{\Delta^{e}\right\}
\end{aligned}
$$

where $E_{i}, A_{i}, I_{i}(i=c, b)$ denoted the Young's modulus, cross-sectional area, and moment of inertia of the constrained layer and the base layer, respectively. $A_{i}$ and $G_{i}(i=s, v)$ were the cross-sectional area and shear modulus of the transition layer and viscoelastic layer, respectively. Here, $G_{i}$ is usually expressed as a plural form. Therefore, through the Eq (10), beam element stiffness could be expressed as:

$$
\begin{aligned}
{\left[K_{b i}\right] } & =\frac{E_{i} I_{i}}{l^{3}} \int_{0}^{1}\left[\frac{\partial^{2} N_{w}}{\partial \xi^{2}}\right]^{T}\left[\frac{\partial^{2} N_{w}}{\partial \xi^{2}}\right] d \xi ; \\
{\left[K_{t i}\right] } & =\frac{E_{i} A_{i}}{l} \int_{0}^{1}\left[\frac{\partial N_{u_{i}}}{\partial \xi}\right]^{T}\left[\frac{\partial N_{u_{i}}}{\partial \xi}\right] d \xi ; \\
{\left[K_{s}\right] } & =G_{s} A_{s} l \int_{0}^{1}\left[N_{\psi}\right]^{T}\left[N_{\psi}\right] d \xi ; \\
{\left[K_{v}\right] } & =G_{v} A_{v} l \int_{0}^{1}\left[N_{\gamma}\right]^{T}\left[N_{\gamma}\right] d \xi ;
\end{aligned}
$$

where $\left[K_{b i}\right],\left[K_{t i}\right]$ were the bending and extensional stiffness matrices of the constrained layer and the base layer, $\left[K_{s}\right],\left[K_{v}\right]$ were the shear stiffness matrix of the viscoelastic layer and the transition layer. So, the total stiffness matrix of the beam element was:

$$
\left[K^{e}\right]=\left[K_{b c}\right]+\left[K_{t c}\right]+\left[K_{b b}\right]+\left[K_{t b}\right]+\left[K_{s}\right]+\left[K_{v}\right] .
$$

\subsection{Beam Element Mass Matrix}

The total kinetic energy of beam element was expressed as: ${ }^{10}$

$$
\begin{aligned}
T=\left[T_{b c}\right]+\left[T_{t c}\right]+ & {\left[T_{b b}\right]+\left[T_{t b}\right] } \\
+ & \left.+T_{b s}\right]+\left[T_{t s}\right]+\left[T_{b v}\right]+\left[T_{t v}\right]
\end{aligned}
$$

where $T_{b c}, T b b$ were the bending kinetic energy and $T_{t c}, T t b$ were the extensional kinetic energy of the constrained layer and the base layer. The variables $T_{b s}, T_{b v}$ were the bending kinetic energy and $T_{t} s, T_{t} v$ were the extensional kinetic energy of the viscoelastic layer and the transition layer. And each component of the total kinetic energy was given by:

$$
\begin{array}{r}
T_{b i}=\frac{1}{2} \rho_{i} A_{i} \int_{0}^{l}\left(\frac{\partial w}{\partial t}\right)^{2} d x=\frac{1}{2}\left\{\dot{\Delta^{e}}\right\}^{T}\left[M_{b i}\right]\left\{\dot{\Delta^{e}}\right\} \\
T_{t i}=\frac{1}{2} \rho_{i} A_{i} \int_{0}^{l}\left(\frac{\partial u_{c}}{\partial t}\right)^{2} d x=\frac{1}{2}\left\{\dot{\Delta^{e}}\right\}^{T}\left[M_{t i}\right]\left\{\dot{\Delta^{e}}\right\} \\
i=(c, b, s, v)
\end{array}
$$

where $\rho_{i}, A_{i}(i=c, b, s, v)$ were the density and crosssectional area of each layer, and the bending mass matrix $\left[M_{b i}\right]$ and the tensile mass matrix $\left[M_{t i}\right]$ of each layer could be expressed as follows:

$$
\begin{gathered}
{\left[M_{b i}\right]=\rho_{i} A_{i} \int_{0}^{l}\left[N_{w}\right]^{T}\left[N_{w}\right] d x=} \\
\rho_{i} A_{i} l \int_{0}^{1}\left[N_{w}\right]^{T}\left[N_{w}\right] d \xi, i=(c, b, s, v)
\end{gathered}
$$

Then, the total element mass matrix could be written as:

$$
\begin{aligned}
{\left[M^{e}\right]=\left[M_{b c}\right]+} & {\left[M_{t c}\right]+\left[M_{b b}\right]+\left[M_{t b}\right] } \\
& +\left[M_{b s}\right]+\left[M_{t s}\right]+\left[M_{b v}\right]+\left[M_{t v}\right] .
\end{aligned}
$$




$$
\begin{aligned}
& N_{w}=\left[\begin{array}{llllllll}
1-3 \xi^{2}+2 \xi^{3} & \left(\xi-2 \xi^{2}+\xi^{3}\right) l & 0 & 0 & 3 \xi^{2}-2 \xi^{3} & \left(-\xi^{2}+\xi^{3}\right) l & 0 & 0
\end{array}\right]
\end{aligned}
$$

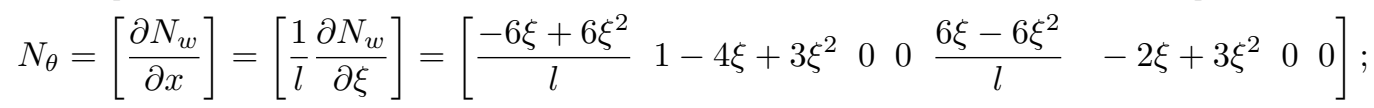

$$
\begin{aligned}
& N_{u_{c}}=\left[\begin{array}{lllllllll}
0 & 0 & 1-\xi & 0 & 0 & 0 & \xi & 0
\end{array}\right] \text {; } \\
& N_{u_{b}}=\left[\begin{array}{lllllllll}
0 & 0 & 0 & 1-\xi & 0 & 0 & 0 & \xi
\end{array}\right] \text {. }
\end{aligned}
$$

\subsection{Dynamic Equation of TCDB}

Based on the Hamilton variational principle, the following equation could be obtained:

$$
\delta \int_{t_{1}}^{t_{2}}(U-T-W) d t=0
$$

where $W=\left\{\Delta^{e}\right\}\left\{R^{e}\right\}$, it denoted the work of all the nonconservative forces which were applied on the transition constrained damping beam. $\left\{R^{e}\right\}$ was the external force; $\delta$ was the variation between the time $t_{1}$ and $t_{2}$. $U, T$ were the total potential energy and kinetic energy, respectively. Substituting the Eqs. (10) and (14) into Eq. (18), the beam element dynamic equations could be determined using the following equation:

$$
M^{e} \ddot{\Delta}^{e}+K^{e} \Delta^{e}=R^{e} .
$$

The above element mass matrix, element stiffness matrix and element external force vector matrix were assembled to obtain total mass matrix $M$, total stiffness matrix $K$ and external force matrix $R$, and then kinetic equation of the TCDB damping beam could be obtained as follows:

$$
M \ddot{\Delta}+K \Delta=R .
$$

\subsection{The Natural Frequency and Loss Factor Calculation}

By solving Eq. (19), the dynamic parameters of TCDB damping beam could be expressed as: ${ }^{18}$

$$
\left([K]-\left(\omega^{*}\right)^{2}[M]\right)\{\Delta\}=0 ;
$$

where $\omega^{*}$ was the angular frequency, and it was always a complex number. At that time, the natural frequency $f$ and the loss factor $\eta$ of the TCDB could be calculated as follows: ${ }^{19}$

$$
\left\{\begin{array}{l}
\eta=\frac{\operatorname{Im}\left[\left(\omega^{*}\right)^{2}\right]}{\operatorname{Re}\left[\left(\omega^{*}\right)^{2}\right]} \\
f=\frac{\sqrt{\operatorname{Re}\left[\left(\omega^{*}\right)^{2}\right]}}{2 \pi}
\end{array}\right.
$$

\section{RESULTS AND DISCUSSION}

\subsection{Comparison with Results in the Literature}

To verify the correctness of the present TCDB finite element

\begin{tabular}{|c|c|c|c|c|c|c|c|}
\hline \multicolumn{4}{|c|}{ Natural frequency $(\mathrm{Hz})$} & \multicolumn{4}{|c|}{ Loss factors } \\
\hline $\begin{array}{c}\text { Mode } \\
\text { No. }\end{array}$ & $\begin{array}{c}\text { Results } \\
\text { from } \\
{[18,19]}\end{array}$ & $\begin{array}{l}\text { Present } \\
\text { method }\end{array}$ & $\begin{array}{c}\text { Error } \\
\%\end{array}$ & $\begin{array}{c}\text { Mode } \\
\text { No. }\end{array}$ & $\begin{array}{c}\text { Results } \\
\text { from } \\
{[18,19]}\end{array}$ & $\begin{array}{l}\text { Present } \\
\text { method }\end{array}$ & $\begin{array}{c}\text { Error } \\
\%\end{array}$ \\
\hline 1 & 64.1 & 64.1 & 0 & 1 & 0.0281 & 0.0281 & 0 \\
\hline 2 & 296.4 & 296.1 & 0.10 & 2 & 0.0242 & 0.0240 & 0.83 \\
\hline 3 & 743.7 & 743.0 & 0.094 & 3 & 0.0154 & 0.0152 & 1.30 \\
\hline 4 & 1393.9 & 1391.8 & 0.15 & 4 & 0.0088 & 0.0086 & 2.27 \\
\hline 5 & 2261.1 & 2258.2 & 0.13 & 5 & 0.0057 & 0.0054 & 5.26 \\
\hline 6 & 3343.6 & 3338.0 & 0.16 & 6 & 0.0039 & 0.0036 & 7.69 \\
\hline
\end{tabular}
model, one example from Daya and Potier-Ferry, and Soni and Bogner was presented. ${ }^{18,19}$ A uniform symmetrical cantilever sandwich beam was taken. Many researchers have analysed this three-layer beam by employing various methods. Here, it
Table 1. Natural frequency and loss factor corresponding to the first six order modes of TCDB.

should be noted that if the material of the transition layer is same as of the viscoelastic layer, it will increase the thickness of viscoelastic layer. Therefore, during the computation of the proposed finite element model of the 4-layer, the total thickness of the transition layer and viscoelastic layer is equal to the thickness of viscoelastic layer in both Daya and Potier-Ferry, and Soni and Bogner, which was $0.127 \mathrm{~mm} .^{18,19}$ The thickness of the transition layer was selected as half of $0.127 \mathrm{~mm}$, which was $0.0635 \mathrm{~mm}$. At the same time, the thickness of the viscoelastic layer was also selected as half of $0.127 \mathrm{~mm}$, which was $0.0635 \mathrm{~mm}$. The material for the base layer and the constrained layer was the same with the modulus of elasticity as $69 \mathrm{Gpa}$, and Poisson ratio was 0.3 , density is $2766 \mathrm{~kg} / \mathrm{m}^{3}$. Both layer thicknesses were $1.524 \mathrm{~mm}$. The shear modulus and loss factor of the viscoelastic layer was $1.794 \mathrm{Mpa}$ and 0.1 . Density and thickness of the viscoelastic layer was $968.1 \mathrm{~kg} / \mathrm{m}^{3}$ and $0.127 \mathrm{~mm}$. The length and width of the beam were $177.8 \mathrm{~mm}$ and $12.7 \mathrm{~mm}$.

The beam was discretized into 40 finite elements, and through successive iteration and using Eq. (22), the natural frequency and loss factor of the beam were calculated. Table 1 shows the comparison between Daya and Potier-Ferry, and Soni and Bogner and present the method for the frequencies and modal loss factors. ${ }^{18,19}$

From Table 1, it could be known that the maximum error of all the six natural frequencies between the analysis solution and the present method was $0.16 \%$, and the average error was $0.11 \%$. At the same time, it could be found that maximum error of the loss factor results was equal to $7.69 \%$, this may have caused by the temperature and frequency effect of viscoelastic material, and the average error of all the modal loss factor was $2.89 \%$. So, there was quite a close map between the results, which showed that the overall analysis could satisfy the engineering requirements, which also verified the correctness of the present TCDB finite element model. 
Table 2. Material and structural parameters of TCDB.

\begin{tabular}{|l|c|c|c|c|}
\hline & $\begin{array}{c}\text { Elastic modulus } \\
(\mathrm{GPa})\end{array}$ & Poisson ratio & $\begin{array}{c}\text { Density } \\
\left(\mathrm{kg} / \mathrm{m}^{3}\right)\end{array}$ & $\begin{array}{c}\text { Thickness } \\
(\mathrm{mm})\end{array}$ \\
\hline Transition layer & 0.29 & 0.35 & 1200 & 0.264 \\
\hline Viscoelastic layer & 0.00029 & 0.45 & 1600 & 0.127 \\
\hline Constrained layer & 72 & 0.33 & 2700 & 0.203 \\
\hline Base layer & 68.9 & 0.33 & 2730 & 2.29 \\
\hline
\end{tabular}

Table 3. Influence of $\eta_{s}$ and $G_{s} / G_{v}$ on the first three-order structural loss factor of TCDB.

\begin{tabular}{||c|c|c|c|c|c|c|c|c||}
\hline \multirow{4}{*}{$\eta_{s}$} & \multicolumn{7}{|c|}{$L g\left(G_{s} / G_{v}\right)$} \\
\cline { 2 - 9 } & 0 & 1 & 1.5 & 2 & 2.5 & 3 & 4 & 5 \\
\hline \multirow{4}{*}{0.1} & 0.0218 & 0.0680 & 0.0758 & 0.0784 & 0.0792 & 0.0795 & 0.0796 & 0.0796 \\
\cline { 2 - 10 } & 0.0041 & 0.0186 & 0.0227 & 0.0243 & 0.0248 & 0.0250 & 0.0251 & 0.0251 \\
\cline { 2 - 9 } & 0.0011 & 0.0063 & 0.0080 & 0.0087 & 0.0089 & 0.0090 & 0.0090 & 0.0090 \\
\hline \multirow{4}{*}{0.3} & 0.0292 & 0.0703 & 0.0766 & 0.0786 & 0.0793 & 0.0795 & 0.0796 & 0.0796 \\
\cline { 2 - 9 } & 0.0055 & 0.0193 & 0.0230 & 0.0244 & 0.0249 & 0.0250 & 0.0251 & 0.0251 \\
\cline { 2 - 9 } & 0.0015 & 0.0065 & 0.0081 & 0.0087 & 0.0089 & 0.0090 & 0.0090 & 0.0090 \\
\hline \multirow{4}{*}{0.8} & 0.0401 & 0.0737 & 0.0777 & 0.0790 & 0.0794 & 0.0795 & 0.0796 & 0.0796 \\
\cline { 2 - 9 } & 0.0076 & 0.0204 & 0.0234 & 0.0245 & 0.0249 & 0.0250 & 0.0251 & 0.0251 \\
\cline { 2 - 9 } & 0.0021 & 0.0069 & 0.0082 & 0.0087 & 0.0089 & 0.0090 & 0.0090 & 0.0090 \\
\cline { 2 - 9 } & 0.0472 & 0.0759 & 0.0785 & 0.0792 & 0.0795 & 0.0796 & 0.0796 & 0.0796 \\
\cline { 2 - 8 } & 0.0089 & 0.0211 & 0.0237 & 0.0246 & 0.0249 & 0.0250 & 0.0251 & 0.0251 \\
\hline
\end{tabular}

\subsection{Influence of the Shear Modulus and Loss Factor of the Transition Layer}

In the present work, a beam with a length of $150 \mathrm{~mm}$ and a width of $11.7 \mathrm{~mm}$ was taken as the research object. The other parameters are given in Table 2. Then the shear modulus, loss factor, and thickness of the transition layer and the thicknesses of the other three layers were discussed about their influences on the frequencies and modal loss factors.

To see the effect of transition layer, it was essential to choose its shear modulus and loss factor reasonably. Table 3 shows the change of the loss factor of TCDB under the different $G_{s} / G_{v}$ ratio when the material loss factor of the transition layer $\left(\eta_{s}\right)$ increases from 0.1 to 0.8 . As can be seen from Table 3, when the $G_{s} / G_{v}$ was relatively small, the material loss factor of transition layer had a more significant effect on the structure loss factor of the TCDB. But with the continuous increase of the $G_{s} / G_{v}$, the influence of $\eta_{s}$ on the structure loss factor gradually weakened, which indicated that the increase of the shear modulus leading to the structure loss factor increase was greater than that of the material loss factor increase. Finally, the structure loss factor gradually became stable and got to an extreme value.

At the same time, by comparing the first three-order structural loss factor in Table 3, it could be found that with the increase of the modal number, the structural loss factor decreased. When the elastic modulus of transition layer was 1000 times bigger than that of viscoelastic layer, even the ratio of $G_{s}$ and $G_{v}$ increased, the structure loss factor gradually tended to a constant value. When the $G_{s} / G_{v}$ ratio was less than 1000 , the structure loss factor could be improved by increasing the material loss factor of transition layer. When the $G_{s} / G_{v}$ ratio was equal to about 1000 , the TCDB could achieve the better effect of energy dissipation without the increase of the material loss factor of transition layer. So, considering the actual effect of energy consumption and economy, it was suggested that the shear modulus value of transition layer should be about
1000 times larger than that of viscoelastic layer.

Table 4 gives the change of the natural frequencies of the structure under the different $G_{s} / G_{v}$ ratio when the material loss factor of the transition layer increased from 0.1 to 0.8 . It could be concluded that the natural frequencies of the whole beam had increased in different degrees with the increase of $G_{s} / G_{v}$, this was because the structure stiffness was proportional to the shear modulus, and the natural frequencies increased with the increase of the structure stiffness. Meanwhile, the material loss factor of the transition layer had a weak influence on the natural frequency of the whole beam. This was due to the shear modulus of the transition layer increasing with the increase of the material loss factor, however, the shear modulus of transition layer was relatively lower than that of the base and the constrained layer. Therefore, it could only bring a small amount of increase of the structure natural frequency of the transition constrained damping beam.

\subsection{Thickness Influence of Transition Layer}

The thickness effects of the transition layer on the natural frequency and the loss factor of the TCDB are shown in Fig. 3. It can be seen from Fig. 3(a), the first-order natural frequency increased and the second and third order natural frequencies decreased when the thickness of transition layer increased. This phenomenon indicated that the effect of the transition layer thickness on the structure natural frequency had a relationship with the modes.

From Fig. 3(b), it could be known that the structural loss factor of TCDB increased when the thickness of the transition layer increased. Further analysis showed that with the increase of the thickness of the transition layer, the first-order modal loss factor gradually slowed down, whereas the second-order modal loss factor tended to increase linearly and an increase in the rate of the third loss factor gradually accelerated. Therefore, an appropriate increase in the thickness of the transition layer was advisable under the condition of meeting the structural size and mass requirements of the whole beam.

\subsection{Thickness influence of Viscoelastic Layer}

The effect of the viscoelastic layer thickness on the natural frequency and the modal loss factor of the TCDB are shown in Fig. 4. It could be seen from Fig. 4(a) that the overall natural frequencies decreased when the viscoelastic layer thickness increased. This was because the viscoelastic layer thickness increase brought about the stiffness and mass increase of the beam at the same time, but the rate of increase for the mass of the beam was bigger than the stiffness of the beam. The overall effect was the decrease of the natural frequency of the whole structure. As it is shown in Fig. 4(b) with the increase of the viscoelastic layer thickness, the loss factors of the second- and third-order decreased at first and then increased, whereas the first modal loss factor got bigger and bigger.

\subsection{Thickness Influence of Constrained Layer}

As it can be seen from Fig. 5(a), the natural frequencies of the whole beam reduced at first and then increased. Further 
Table 4. Influence of $\eta_{s}$ and $G_{s} / G_{v}$ on the first three-order natural frequency of TCDB.

\begin{tabular}{|c|c|c|c|c|c|c|c|c|c|}
\hline \multirow[b]{2}{*}{$\eta_{s}$} & \multicolumn{9}{|c|}{$L g\left(G_{s} / G_{v}\right)$} \\
\hline & 0 & 0.5 & 1 & 1.5 & 2 & 2.5 & 3 & 4 & 5 \\
\hline \multirow{3}{*}{0.1} & 79.21 & 81.15 & 82.66 & 83.40 & 83.67 & 83.77 & 83.79 & 83.81 & 83.81 \\
\hline & 482.12 & 484.30 & 486.09 & 486.99 & 487.33 & 487.45 & 487.49 & 487.50 & 487.50 \\
\hline & 1344.17 & 1345.82 & 1347.18 & 1347.87 & 1348.1 & 1348.22 & 1348.25 & 1348.26 & 1348.26 \\
\hline \multirow{3}{*}{0.3} & 79.25 & 81.24 & 82.72 & 83.43 & 83.68 & 83.77 & 83.80 & 83.80 & 83.81 \\
\hline & 482.14 & 484.35 & 486.12 & 487.00 & 487.34 & 487.45 & 487.49 & 487.49 & 487.50 \\
\hline & 1344.17 & 1345.82 & 1347.18 & 1347.87 & 1348.1 & 1348.22 & 1348.25 & 1348.25 & 1348.26 \\
\hline \multirow{3}{*}{0.6} & 79.34 & 81.38 & 82.82 & 83.46 & 83.70 & 83.77 & 83.80 & 83.81 & 83.81 \\
\hline & 482.18 & 484.42 & 486.17 & 487.03 & 487.35 & 487.45 & $\begin{array}{l}487.49 \\
\end{array}$ & 487.50 & 487.50 \\
\hline & 1344.16 & 1345.82 & 1347.18 & 1347.87 & 1348.1 & 1348.22 & 1348.25 & 1348.26 & 1348.26 \\
\hline \multirow{3}{*}{0.8} & 79.42 & 81.48 & 82.88 & 83.49 & 83.70 & 83.78 & 83.80 & 83.81 & 83.81 \\
\hline & 482.21 & 484.47 & 486.21 & 487.04 & 487.35 & 487.45 & 487.49 & 487.50 & 487.50 \\
\hline & 1344.16 & 1345.81 & 1347.18 & $\begin{array}{l}1347.87 \\
\end{array}$ & $\begin{array}{ll}1348.1 \\
\end{array}$ & 1348.22 & 1348.25 & 1348.26 & 1348.26 \\
\hline
\end{tabular}

analysis showed that the first mode frequency underwent a very small decline, and then continued to increase. The second- and third-order frequencies also experienced a brief decline and then they increased, but the rate of increasing of the latter two frequencies was faster. In Fig. 5(b), all of the loss factors of the TCDB first increased and then decreased when the constrained layer thickness increased. The first modal loss factor reached the maximum when the thickness ratio of the constrained layer and the base layer was equal to 0.8 .

\subsection{Thickness Influence of Base Layer}

Figure 6 shows the influence of the base layer thickness on the natural frequency and loss factor of TCDB. From Fig. 6(a), it could be seen that all of the natural frequencies increased with the increase of the thickness of the base layer. That was because when the base layer's thickness increased, the stiffness and mass of the whole transition constrained damping beam increased, but the stiffness increase was greater than that of the mass. Therefore, the frequencies displayed a trend of increase.

At the same time, the structure loss factor of the transition constrained damping beam decreased rapidly with the increase of the thickness of the base layer. This was because the attenuation of vibration energy became more difficult when the base layer thickness increased under the same conditions. Therefore, when the base layer thickness was chosen, it was advisable to reduce its thickness under the condition of meeting the stiffness and strength of the whole structure. From the point of view of engineering design, although the damping treatment was a kind of post-damping method, the results about the discussion of the base layer thickness in this paper can provide some reference for the initial thickness choice.

\subsection{Relationship Between Structural Loss Factor and Frequency}

Figure 7 gives the structural loss factor variation with respect to frequencies. In contrast to Figs. 3-6, it provides a wider frequency range. From the Fig. 7(a), it could be known that in the given frequency range (the first six mode frequency), the variation was different as to the different thicknesses of the transition layer. When the thickness of the transition layer was selected as $0.1,0.15$, and 0.2 , the loss factors decreased at first and then increased at a different rate. When the thickness of the transition layer was equal to 0.25 and 0.3 , the loss factors decreased all the time. At the same time, from the Fig. 7(a), it could be concluded that in the low frequency range, with the thickness increase of the transition layer, the loss factors grew. However, in the higher frequency range, this phenomenon took a change. No what matter the thickness of the transition layer was, the effect of vibration-reducing was better in the low frequency range than that of the high frequency. Therefore, the changes of loss factor on frequency were not monotonous.

Similarly, from the Fig. 7(b), we could know that when the thickness of the viscoelastic layer was comparatively big (e.g. 0.50), the loss factors first got smaller and then increased again at the given frequency range. In addition, when the thickness of the viscoelastic layer was comparatively small (e.g. 0.10), the loss factors of the TCDB got smaller and smaller. On the other hand, the first-order loss factor increased as the thickness of the viscoelastic layer increased. But in the higher frequency range (e.g. the fourth, fifth and sixth natural frequency), the structural loss factors decreased first and then increased rapidly. The larger the thickness, the more obvious this phenomenon.

From the Fig. 7(c), it is easy to see that the damping performance of the TCDB at the lower frequency is better than that of the higher frequency. The loss factor of the TCDB changed continuously with the change of frequency, so long as the frequency was equal or close, the loss factor was equal or close, too. In addition, the effect of the thickness of the constrained layer on the structural loss factor was different in the whole frequency range.

At last, from the Fig. 7(d), the structure loss factors of the TCDB got smaller and smaller with the increase of frequency.

\section{CONCLUSIONS}

In this paper, a finite element model of TCDB is established to calculate a structure's natural frequency and loss factor according to the first shear deformation theory, and the vibration and damping characteristic are analysed. The specific results are given as follows:

1. When the transition layer material is chosen, its shear modulus is advisable to be 1000 times greater than that of the viscoelastic layer and its material loss factor is as big as possible. At the same time, the increase of the thickness of the transition layer is beneficial under the condition of meeting the size and mass requirement of the whole transition constrained damping beam. 


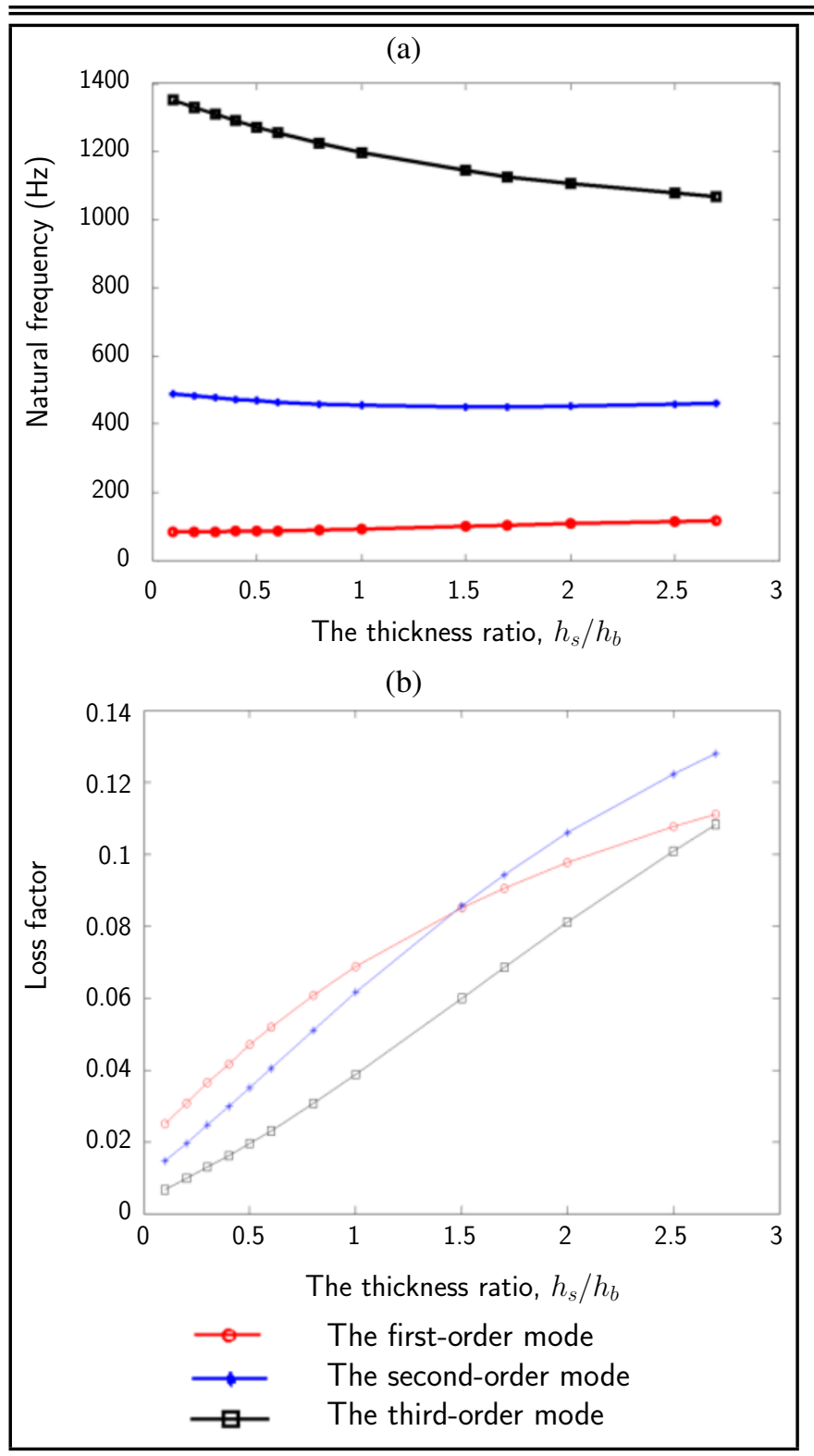

Figure 3. Influence of transition layer thickness on (a) natural frequency and (b) loss factor.

2. When the viscoelastic layer thickness increases, the natural frequencies tend to decrease and the structure loss factors decrease at first and then increase. As the constrained layer thickness increases, the natural frequencies decrease at first and then increase, and the structure loss factors increase at first and then decrease. For the base layer, its thickness may be reduced appropriately under the condition of meeting the stiffness and strength of the whole structure.

\section{FOUNDING}

This project is sponsored by the fund for Shanxi "1331" project Key Subjects Construction (1331KSC), the Natural Science Foundation of Shanxi Province (Grant No. 201701D121071), and National Natural Science Foundation of China (Grant No. 51805347, 51405323).

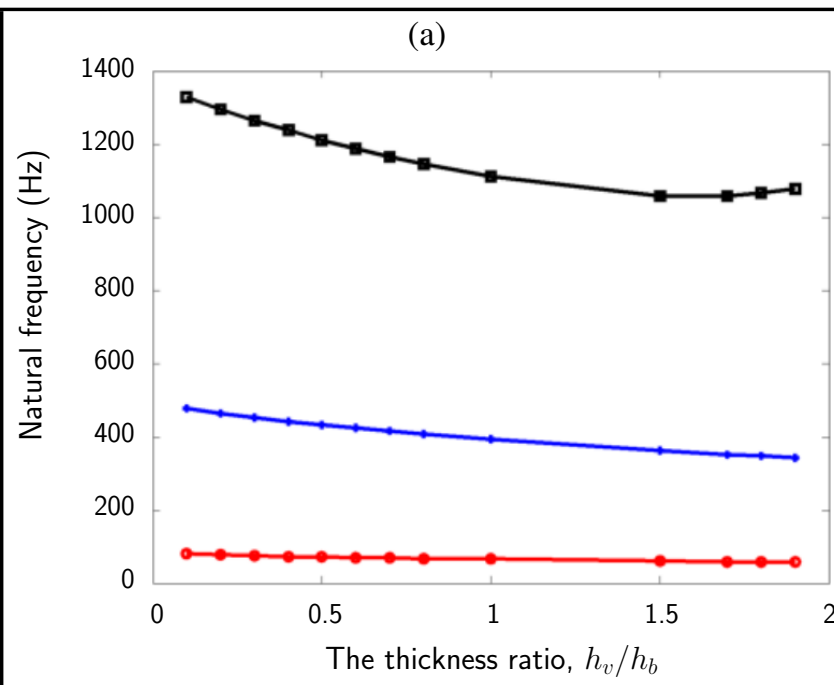

(b)

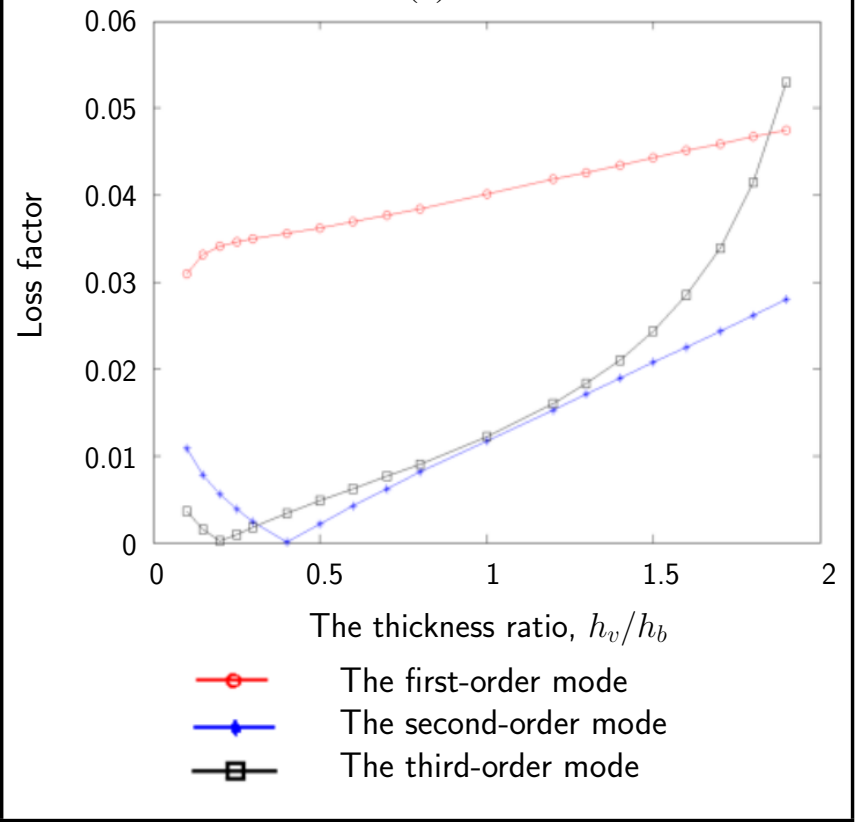

Figure 4. Influence of viscoelastic layer thickness on (a) natural frequency and (b) loss factor.

\section{REFERENCES}

1 Huang Z. C., Qin C. Y., Chu F. L. Transverse vibration of viscoelastic sandwich beams based on the compression dissipating energy assumption, $J$. Vibration and Shock., 35 (10), 185-191, (2016). https://dx.doi.org/10.13465/j.cnki.jvs.2016.10.030

2 Lepoittevin G., Kress, G. Optimization of segmented constrained layer damping with mathematical programming using strain energy analysis and modal data, J. Material Design., 31 (1), 14-24, (2011). https://dx.doi.org/10.1016/j.matdes.2009.07.026

3 Kumar R. S., Ray, M. C. Active constrained damping of smart laminated structure sandwich plates using 1-3 piezoelectric structures, J. International Journal of Mechanics and Material in Design., 8 (3), 197-218, (2012). https://dx.doi.org/10.1007/s10999-012-9201-y

4 Yan B. J., Zhang W. J., Li Z. L., Sun, D. G. Distributed parameter transfer function method for dynamic re- 


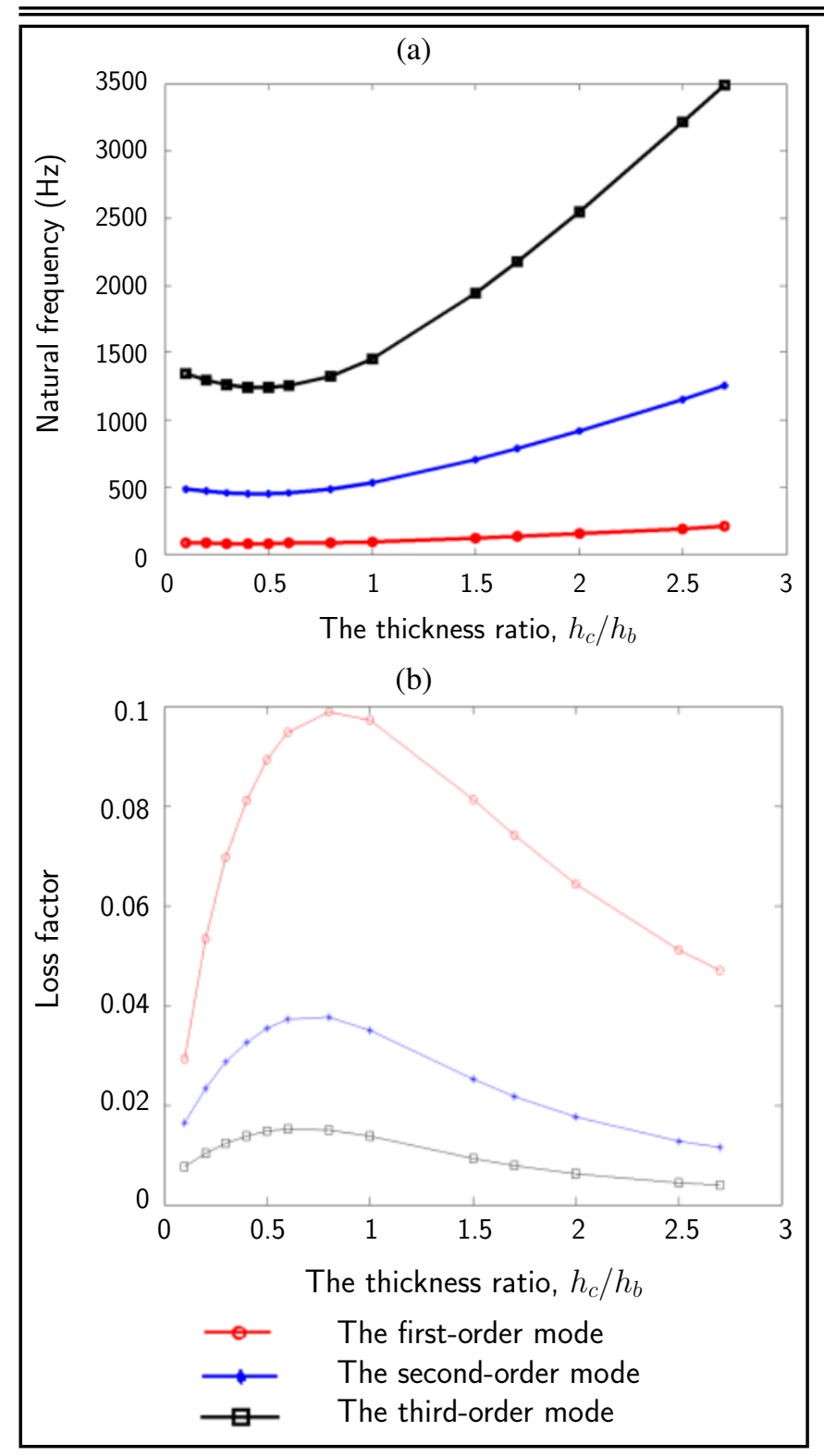

Figure 5. Influence of constrained layer thickness on (a) natural frequency and (b) loss factor.

sponse of a constrained damping structure with transition layer, J. Vibration and Shock., 35 (5), 186-190, (2016). https://dx.doi.org/10.13465/j.cnki.jvs.2016.05.030

5 Li E. Q., Tang G. J., Lei Y. J., Li, D. K. Dynamic analysis of constrained layer damping plate by the transfer function method, J. National University of Defense Technology., 30 (1), 5-9, (2008). https://dx.doi.org/10.3969/j.issn.10012486.2008.01.002

6 Kerwin, E. M. Damping of flexural waves by a constrained viscoelastic layer, J. The Acoustical Society of America., 31 (7), 952-962, (1959). https://dx.doi.org/10.1121/1.1907821

7 Ditaranto, R. A. Theory of vibratory bending for elastic and viscoelastic layered finite-length beams, J. Applied Mechanics, 32 (4), 881-886, (1965). https://dx.doi.org/10.1115/1.3627330

${ }^{8}$ Rao D. K. Frequency and loss factors of sandwich beams under various boundary conditions, J. Me- (a)

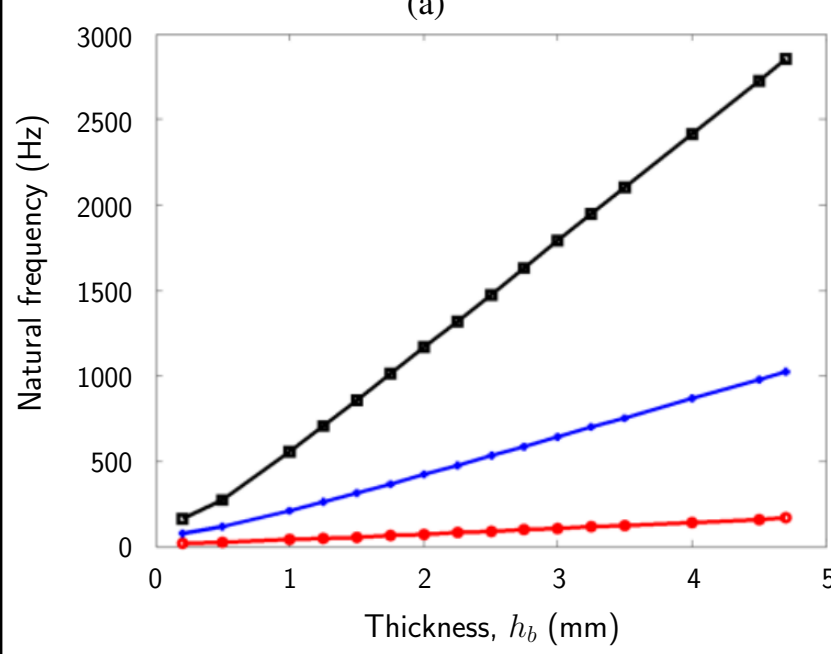

(b)

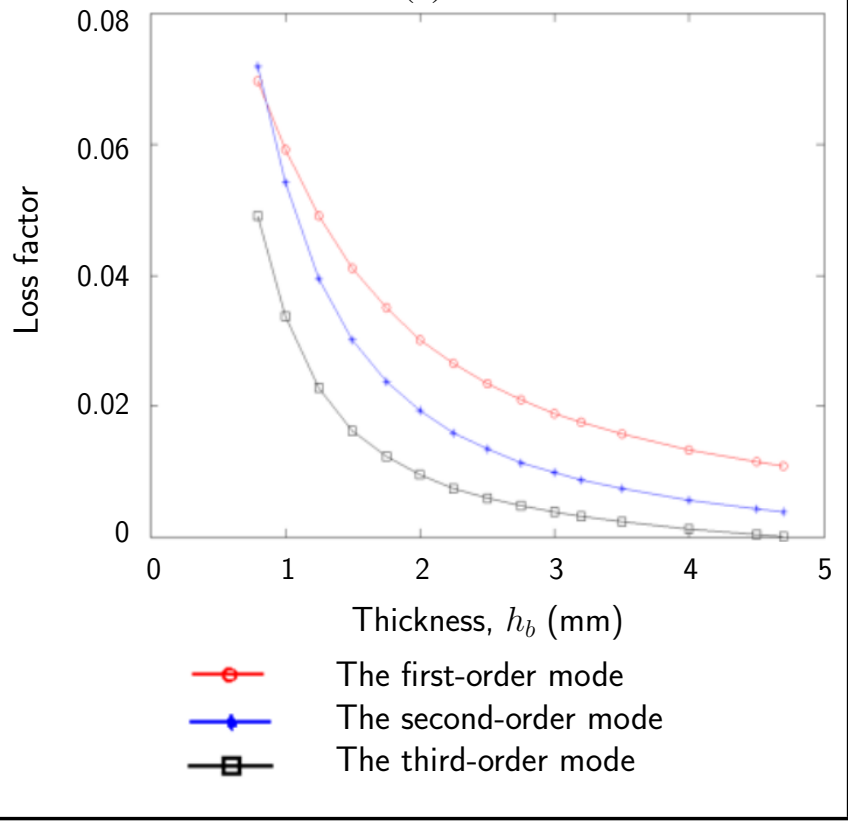

Figure 6. Influence of base layer thickness on (a) natural frequency and (b) loss factor.

chanical Engineering Science., 20 (5), 271-282, (1978). https://dx.doi.org/10.1243/JMES_JOUR_1978_020_047_02

9 Tang G. J., Li E. Q., Li D. K., Lei, Y. J. Semianalytical dynamics of constrained layer damping plate, J. Chinese Journal of Solid Mechanics., 29 (2), 149156, (2008). https://dx.doi.org/10.19636/j.cnki.cjsm421250/o3.2008.02.006

10 Huang Z. C., Qin C. Y., Chu F. L. Vibration and damping characteristics analysis of viscoelastic sandwich beams based on the shear dissipating energy assumption, J, Vibration and Shock., 34 (7), 183-191, (2015). https://dx.doi.org/10.13465/j.cnki.jvs.2015.07.029

11 Johnson C. D., Kienholz D. A. Finite element prediction of damping in structures with constrained viscoelastic layers, J. AIAA., 20 (20), 1284-1290, (1982). https://dx.doi.org/10.2514/3.51190

12 Sainsbury M. G., Zhang Q. J. The Galerkin element method applied to the vibration of damped sandwich 


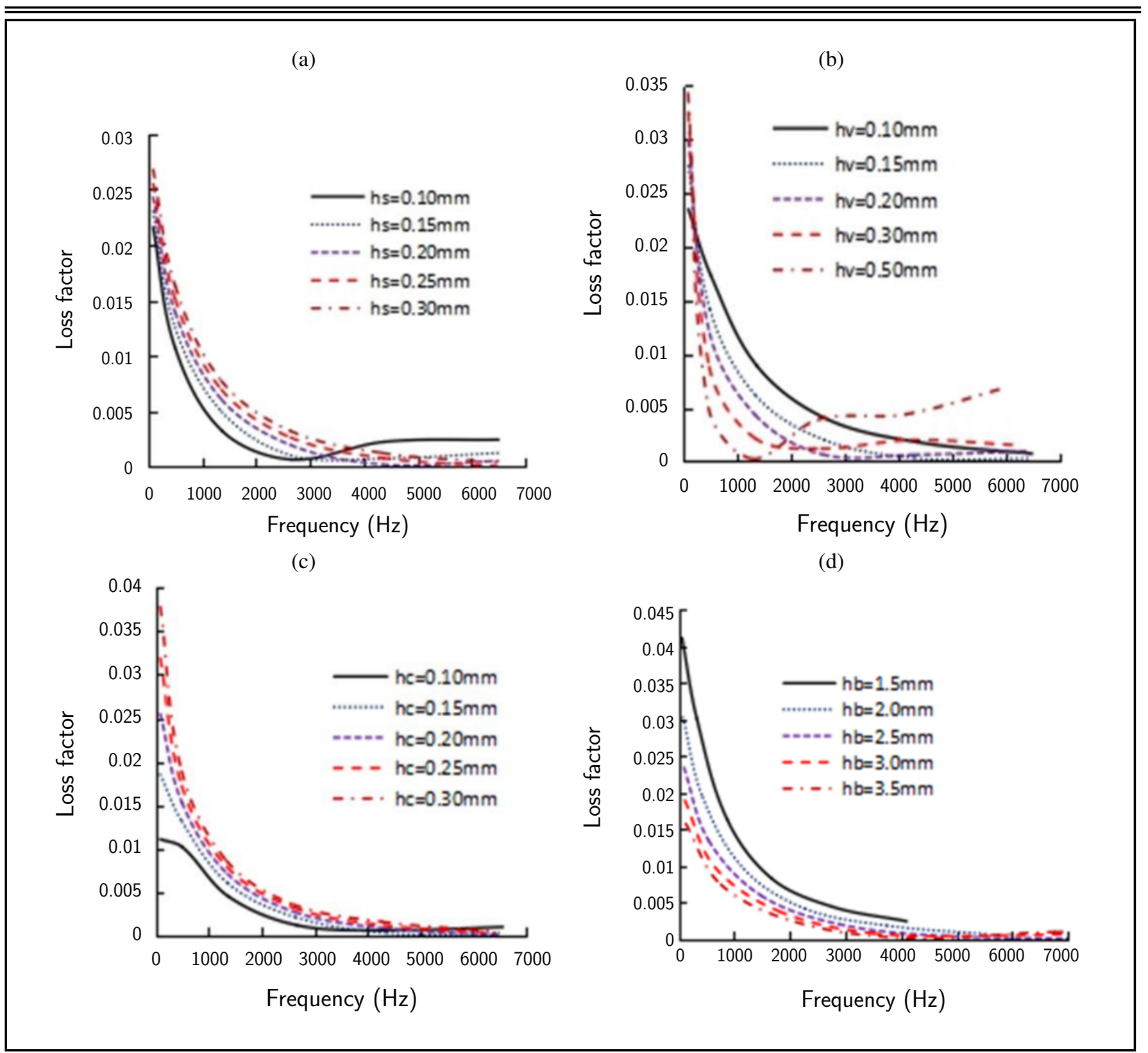

Figure 7. Relationship between the structural loss factors and frequencies.

beams, J. Computer \& Structures., 71 (3), 239-256, (1999). https://dx.doi.org/10.1016/S0045-7949(98)00242-9

13 Deng N. C., Zhou Z. Z., Du H. J. A finite element dynamic analysis of constrained plates, J. Vibration Engineering., 16 (4), 489-492, (2003). https://dx.doi.org/10.3969/j.issn.1004-4523.2003.04.021

${ }^{14}$ Zhang W. J., Sun D. G., Yan B. J., Li, Z. L., Sun, B. Complex stiffness method of loss factor for constrained damping structure with transition layer, $J . B a$ sic Science and Engineering., 24 (6), 1296-1307, (2016). https://dx.doi.org/10.16058/j.issn.1005-0930.2016.06.020

15 Baber T. T., Maddox A. R., Orazco C. E. Finite element model for harmonically excited viscoelastic sandwich beams, J. Computers \& Structure., 66 (1),105-113, (1998). https://dx.doi.org/10.1016/S0045-7949(97)00046-1

16 Chaudry, A. H. Passive stand-off layer damping treatment: theory and experiments, University of Maryland, (2006).
17 Kumar S., Kumar R., Ralcesh S. Enhanced ACLD treatment using stand-off-layer: FEM based design and experimental vibration analysis, J. Applied Acoustics., 72 (11), 856-872, (2011). https://dx.doi.org/10.1016/j.apacoust.2011.05.010

18 Daya E. M., Potier-Ferry, M. A numerical method for nonlinear eigenvalue problems application to vibrations of viscoelastic structures, J. Computer and Structure., 79 (5), 533-541, (2001). https://dx.doi.org/10.1016/S00457949(00)00151-6

19 Soni, M. L. and Bogner, F. K. Finite element analysis of viscoelastically damped sandwich structures, J. Shock Vibrat. Bull, 55 (1), 97-109, (1981). https://dx.doi.org/10.2514/3.51127 